\title{
Soft Gluon Effects in Four-Parton Hard-Scattering Processes
}

\author{
Alessandro Torre* ${ }^{* \dagger}$ \\ Institut für Theoretische Physik, Universität Zürich, CH-8057 Zürich, Switzerland \\ E-mail: atorre@physik.uzh.ch
}

We consider the threshold limit of the one-particle inclusive cross section, and we compute the logarithmically-enhanced terms at next-to-leading-order in perturbative QCD. We keep control of the colour-correlation effects arising from the interference of large-angle soft-radiation and the hard process-dependent colour amplitude. We present the all-order soft-gluon resummation of the logarithmic terms and we give its explicit expression up to next-to-leading logarithmic accuracy. The factorized form of our fixed-order computation allows us to extract the one-loop hard-virtual amplitude entering our resummation formula. This paves the way to the explicit extension of the resummation formula beyond next-to-leading logarithmic accuracy.

11th International Symposium on Radiative Corrections (Applications of Quantum Field Theory to Phenomenology) (RADCOR 2013),

22-27 September 2013

Lumley Castle Hotel, Durham, UK

\footnotetext{
* Speaker.

${ }^{\dagger}$ Work done in collaboration with Stefano Catani and Massimiliano Grazzini.
} 


\section{Introduction}

Hard-scattering processes described by perturbative QCD are affected by soft and collinear enhancements, when the available phase space is constrained by specific, observable-dependent boundaries. By approaching these boundaries, real radiation is kinematically inhibited. The imbalance with virtual radiation leads to large logarithmic terms, that are singular at the kinematical boundary. They can invalidate the (quantitative) reliability of the order-by-order perturbative expansion, already at the relative next-to-leading order. A reliable evaluation of any cross-section in the near-threshold region requires the all-order resummation of these logarithms. A distinction can be made between energy (invariant-mass) and transverse-momentum thresholds. Here we focus on invariant-mass thresholds and the so called threshold-resummation $[1,2]$.

In the context of hadron-hadron collisions, a class of soft-gluon sensitive observables is represented by inclusive hard-scattering cross sections in kinematical configurations that are close to the (partonic) threshold. Typical examples are the cross sections for the production of Drell-Yan lepton pairs and Higgs bosons and explicit resummed results have been obtained up to next-tonext-to-leading logarithmic (NNLL) accuracy $[3,4]$. In the case of the hard scattering of three and four (or more) coloured partons, soft-gluon dynamics leads to non-trivial colour correlations and colour coherence effects. The general soft-gluon resummation formalism for inclusive QCD cross sections was developed in $[5,6,7,8,9,10]$. Some examples of relevant processes with three or four partons at the Born level in QCD are the direct production of prompt photons [8, 9, 11, 12], vector boson [13] and Higgs boson [15] production at high transverse momentum, production of heavy quarks [5, 6, 8, 16] and coloured supersymmetric particles (Ref. [17] and references therein) at hadron colliders, single top-quark production [18], jet [19] and dihadron [20] production, and single-hadron inclusive production in hadronic collisions [21].

In the following we consider the single-hadron inclusive cross section at fixed rapidity of the observed hadron and we summarise the results of [22].

\section{NLO results near the partonic threshold}

We consider the inclusive hard-scattering reaction $h_{1}\left(P_{1}\right)+h_{2}\left(P_{2}\right) \rightarrow h_{3}\left(P_{3}\right)+X$, where the collision of the two hadrons $h_{1}$ and $h_{2}$ with momenta $P_{1}$ and $P_{2}$, respectively, produces the hadron $h_{3}$ with momentum $P_{3}$ accompanied by an arbitrary and undetected final state $X$. According to the QCD factorization theorem the corresponding cross section is given by

$$
\begin{aligned}
E_{3} \frac{d \sigma_{h_{3}}}{d^{3} \mathbf{P}_{3}}\left(P_{1}, P_{2}, P_{3}\right) & =\sum_{a_{1}, a_{2}, a_{3}} \int_{0}^{1} d x_{1} \int_{0}^{1} d x_{2} \int_{0}^{1} \frac{d x_{3}}{x_{3}^{2}} f_{a_{1} / h_{1}}\left(x_{1}, \mu_{F}\right) f_{a_{2} / h_{2}}\left(x_{2}, \mu_{F}\right) d_{a_{3} / h_{3}}\left(x_{3}, \mu_{f}\right) \\
& \times p_{3}^{0} \frac{d \hat{\sigma}_{a_{1} a_{2} \rightarrow a_{3}}}{d^{3} \mathbf{p}_{3}}\left(x_{1} P_{1}, x_{2} P_{2}, P_{3} / x_{3} ; \mu_{F}, \mu_{f}\right)
\end{aligned}
$$

where $f_{a / h}\left(x, \mu_{F}\right)$ is the parton density of the colliding hadron evaluated at the factorization scale $\mu_{F}$, and $d_{a / H_{3}}\left(x, \mu_{f}\right)$ is the fragmentation function of the parton $a$ into the hadron $H_{3}$ at the factorization scale $\mu_{f}$, as defined in the $\overline{\mathrm{MS}}$ factorization scheme. The last factor, $d \hat{\sigma}_{a_{1} a_{2} \rightarrow a_{3}}\left(p_{1}, p_{2}, p_{3}\right)$ is the inclusive cross section for the inelastic scattering $a_{1}\left(p_{1}\right)+a_{2}\left(p_{2}\right) \rightarrow a_{3}\left(p_{3}\right)+X$, which, throughout the paper, is always treated with massless partons (kinematics). 
The NLO calculation for this process was carried out in analytical form, and it is presented $[23,24,25]$ in terms of the independent kinematical variables $s, v$ and $w$, which are related to the customary Mandelstam variables $s, t, u$ through the definition $v \equiv 1+t / s, w \equiv-u /(s+t)$ with the corresponding phase-space boundaries $s \geq 0,1 \geq v \geq 0,1 \geq w \geq 0$. Using these variables, the partonic cross section in Eqs. (2.1) can be written as

$$
\begin{aligned}
p_{3}^{0} \frac{d \hat{\sigma}}{d^{3} \mathbf{p}_{3}}\left(p_{1}, p_{2}, p_{3} ; \mu_{F}, \mu_{f}\right)=\frac{\alpha_{\mathrm{S}}^{2}\left(\mu_{R}^{2}\right)}{\pi s} & {\left[\frac{1}{v} \frac{d \hat{\sigma}^{(0)}(s, v)}{d v} \delta(1-w)\right.} \\
& \left.+\frac{\alpha_{\mathrm{S}}\left(\mu_{R}^{2}\right)}{2 \pi} \frac{1}{v s} \mathscr{C}^{(1)}\left(s, v, w ; \mu_{R}, \mu_{F}, \mu_{f}\right)+\mathscr{O}\left(\alpha_{\mathrm{S}}^{2}\right)\right],
\end{aligned}
$$

where the flavour indices are left understood. The Born-level term in Eq. (2.2) has a sharp integrable singularity at $w=1$. At the LO, the system $X$ is formed by a single massless parton $a_{4}\left(p_{4}\right)$ and, therefore, $s_{X}=p_{4}^{2}$ exactly vanishes thus leading to the factor $\delta(1-w)$ in Eq. (2.2). At higher perturbative orders, the LO singularity is enhanced by logarithmic terms of the type $\ln (1-w)$. The enhancement has a dynamical origin, and it is produced by soft-gluon radiation.

The presence of logarithmically-enhanced terms is evident from the known NLO result. The structure of the NLO term $\mathscr{C}^{(1)}$ in Eq. (2.2) is customarily written (see, e.g., Eqs. (10) and (22) in Ref. [25]) in the following form:

$$
\begin{aligned}
\mathscr{C}^{(1)}\left(s, v, w ; \mu_{R}, \mu_{F}, \mu_{f}\right) & =\mathscr{C}_{3}(v)\left(\frac{\ln (1-w)}{1-w}\right)_{+}+\mathscr{C}_{2}\left(v ; s, \mu_{F}, \mu_{f}\right)\left(\frac{1}{1-w}\right)_{+} \\
& +\mathscr{C}_{1}\left(v ; s, \mu_{R}, \mu_{F}, \mu_{f}\right) \delta(1-w)+\mathscr{C}_{0}\left(1-w, v ; s, \mu_{R}, \mu_{F}, \mu_{f}\right) .
\end{aligned}
$$

The last term on the right-hand side is a non-singular function of $w$ in the limit $w \rightarrow 1$, namely, $\mathscr{C}_{0}(1-w, v)=\mathscr{O}\left((1-w)^{0}\right)$ The functions $\mathscr{C}_{3}, \mathscr{C}_{2}$ and $\mathscr{C}_{1}$ do not depend on $w$, and they multiply functions of $w$ that are singular (and logarithmically-enhanced) at $w \rightarrow 1$. These singular functions are expressed by $\delta(1-w)$ and customary 'plus-distributions', $\left[\left(\ln ^{k}(1-w)\right) /(1-w)\right]_{+}$, defined over the range $1 \geq w \geq 0$.

Due to unitarity, the singular structure of virtual corrections must match the singular structure of real corrections at the same perturbative order. This ensures the cancellation of poles and, at the same time, fixes the coefficients of the threshold logarithms. In fact, functions $\mathscr{C}_{3}$ and $\mathscr{C}_{2}$ can be determined by the (IR) divergent terms, that have a process-independent structure. The constant (i.e. non-logarithmic) term $\mathscr{C}_{1}$ contains instead an amount of process-dependent information that can be extracted from the finite (IR-subtracted) one-loop amplitude of the elastic process. More importantly, its behaviour is controlled by non trivial colour-correlations effects, which play a role also beyond the NLO, thanks to real-virtual interference. In order to derive an all-order resummed cross-section for this process, one needs to keep colour-correlations under control, at each order in perturbation theory (see Sect. 3). Explicitely, we need the expression of the one-loop amplitude as a colour-space vector. The available NLO QCD calculation of the single-hadron inclusive crosssection $[23,24,25]$ corresponds to projections of the colour amplitude on the available colour (flavour) channels.

A new calculation of the NLO cross-section at threshold is presented in [22]. The final result of $\mathscr{C}_{1}$ is given in terms of abstract colour operators [26, 27], that describe radiation off a generic massless parton. The key ingredient is the description of soft real radiation via the process-independent 
eikonal approximation, which makes the computation of the colour-space amplitude possible. The available phase-space of the recoiling jet allows non-soft collinear splitting. This is taken into account by an appropriate extension of the eikonal approximation, according to the Altarelli-Parisi splitting kernels. The IR corrections are then complemented by the $\overline{\mathrm{MS}}$ collinear counterterms of the initial-state parton densities and the fragmentation function of the observed hadron.

Using the one-loop virtual contributions from Ref. [28], we have verified that we correctly reproduce the NLO results of Ref. [25] for the NLO coefficient $\mathscr{C}^{(1)}$ of the various partonic channels. An additional check is given by the case in which the observed parton is replaced by a photon. Using the one-loop virtual contribution for the process $g q \rightarrow \gamma q$ [30] and its crossing-related channels, we have explicitly verified that our result correctly reproduces the NLO coefficient of the cross section for prompt-photon production [31, 32].

\section{All-order soft-gluon resummation}

Soft-gluon resummation up to NLL to the partonic cross section was performed in Ref. [21], after integration over the rapidity of the observed hadron. The quantitative effect of resummation is rather large, especially in the kinematical configurations that are encountered in experiments at the typical energies of fixed-target collisions. We use the formalism of Ref. [10], and we present the soft-gluon resummation formula that controls the logarithmic contributions to the rapidity distribution of the transverse-momentum Using our general expression of the NLO cross section, we determine the one-loop hard-virtual amplitude that enters into the colour-space factorization structure of the resummation formula.

To discuss the all-order resummation, we introduce the independent kinematical variables

$$
x_{\omega}=-\frac{u+t}{s}, \quad r=\frac{u}{t}, \quad p_{T}^{2}=\frac{u t}{s},
$$

with phase-space boundaries $1 \geq x_{\omega} \geq 0, r \geq 0, p_{T}^{2} \geq 0 . p_{T}$ is the transverse momentum of the observed parton $a_{3}$. In the centre-of-mass frame of the partonic collision, the variable $x_{\omega}$ is the energy fraction of the parton $a_{3}$ and $r$ is related to its scattering angle. The near-threshold corresponds to the region where $x_{\omega} \rightarrow 1$, at fixed values of $p_{T}$ and $r$. Using these variables,

$$
p_{3}^{0} \frac{d \hat{\sigma}_{a_{1} a_{2} \rightarrow a_{3}}}{d^{3} \mathbf{p}_{3}}=\frac{1}{s} \frac{\left.\overline{\mid \mathscr{M}_{a_{1} a_{2} a_{3} a_{4}}^{(0)}}\right|^{2}}{16 \pi^{2} s} \Sigma_{a_{1} a_{2} \rightarrow a_{3}}\left(x_{\omega}, r ; p_{T}^{2}, \mu_{F}, \mu_{f}\right) .
$$

The QCD radiative corrections are embodied in the function $\Sigma_{a_{1} a_{2} \rightarrow a_{3}}$. We perform resummation in Mellin space $[1,2]$. The Mellin space $N$-moments $\Sigma_{N}$ of the function $\Sigma\left(x_{\omega}\right)$ is

$$
\Sigma_{a_{1} a_{2} \rightarrow a_{3}, N}(r) \equiv \int_{0}^{1} d x_{\omega} x_{\omega}^{N-1} \Sigma_{a_{1} a_{2} \rightarrow a_{3}}\left(x_{\omega}\right)=\Sigma_{a_{1} a_{2} \rightarrow a_{3} a_{4}, N}^{\mathrm{res}}(r)+\mathscr{O}(1 / N),
$$

where $\Sigma_{N}^{\text {res }}$ includes the all-order resummation of the $\ln N$ terms. Contributions of $\mathscr{O}(1 / N)$ that are subdominant in the near-threshold limit are neglected. The all-order expression of $\Sigma_{N}^{\text {res }}$ can be derived using the techniques of Ref. [10], and it reads

$$
\Sigma_{a_{1} a_{2} \rightarrow a_{3} a_{4}, N}^{\mathrm{ras}}\left(r ; p_{T}^{2}, \mu_{F}, \mu_{f}\right)=\left[\prod_{i=1,2,3} \Delta_{a_{i}, N_{i}}\left(Q_{i}^{2} ; \mu_{F i}^{2}\right)\right] J_{a_{4}, N_{4}}\left(Q_{4}^{2}\right) \frac{\left\langle\mathscr{M}_{H}\left|\Delta_{N}^{(\mathrm{int})}\left(r ; p_{T}^{2}\right)\right| \mathscr{M}_{H}\right\rangle}{\left|\mathscr{M}^{(0)}\right|^{2}} .
$$


The three radiative factors $\Delta_{a_{i}, N}(i=1,2,3)$ embody soft-gluon radiation from the triggered partons of the partonic process. The jet function $J_{a_{4}, N_{4}}$ includes soft and collinear radiation from the parton $a_{4}$ that recoils against the observed parton $a_{3}$ in the tree-level elastic scattering process. We have

$$
\begin{aligned}
& \Delta_{a, N}\left(Q^{2} ; \mu^{2}\right)=\exp \left\{\int_{0}^{1} d z \frac{z^{N-1}-1}{1-z} \int_{\mu^{2}}^{(1-z)^{2} Q^{2}} \frac{d q^{2}}{q^{2}} A_{a}\left(\alpha_{\mathrm{S}}\left(q^{2}\right)\right)\right\}, \\
& J_{a, N}\left(Q^{2}\right)=\exp \left\{\int_{0}^{1} d z \frac{z^{N-1}-1}{1-z}\left[\int_{(1-z)^{2} Q^{2}}^{(1-z) Q^{2}} \frac{d q^{2}}{q^{2}} A_{a}\left(\alpha_{\mathrm{S}}\left(q^{2}\right)\right)+\frac{1}{2} B_{a}\left(\alpha_{\mathrm{S}}\left((1-z) Q^{2}\right)\right)\right]\right\},
\end{aligned}
$$

where $A_{a}\left(\alpha_{\mathrm{S}}\right)$ and $B_{a}\left(\alpha_{\mathrm{S}}\right)$ are perturbative functions. Their lower-order coefficients are

$$
A_{a}^{(1)}=C_{a}, \quad A_{a}^{(2)}=\frac{1}{2} C_{a} K, \quad K=\left[C_{A}\left(\frac{67}{18}-\frac{\pi^{2}}{6}\right)-\frac{5}{9} n_{F}\right], \quad B_{a}^{(1)}=-\gamma_{a},
$$

where

$$
\gamma_{q}=\gamma_{\bar{q}}=\frac{3}{2} C_{F}, \quad \gamma_{g}=\frac{11}{6} C_{A}-\frac{1}{3} n_{F} .
$$

The values of $N_{i}$ and $Q_{i}^{2}$ in the argument of the radiative factors depend on $r, p_{T}^{2}, N$ and involves some degree of arbitrariness. The last factor in the right-hand side of Eq. (3.4) has a factorized structure in colour space, and it includes all the colour correlation effects. The colour-space radiative factor $\Delta_{N}^{(\text {int })}$ embodies all the quantum-interference effects produced by soft-gluon radiation at large angles and reads [10]

$$
\begin{aligned}
\Delta_{N}^{(\mathrm{int})}\left(r ; p_{T}^{2}\right) & =V_{N}^{\dagger}\left(r ; p_{T}^{2}\right) V_{N}\left(r ; p_{T}^{2}\right), \\
V_{N}\left(r ; p_{T}^{2}\right) & =P_{z} \exp \left\{\int_{0}^{1} d z \frac{z^{N-1}-1}{1-z} \Gamma\left(\alpha_{\mathrm{S}}\left((1-z)^{2} p_{T}^{2}\right) ; r\right)\right\} .
\end{aligned}
$$

The operator $P_{z}$ denotes $z$-ordering in the expansion of the exponential matrix. The soft-gluon anomalous dimension $\Gamma\left(\alpha_{\mathbb{S}} ; r\right)$ is a colour space matrix, with perturbative expansion

$$
\Gamma\left(\alpha_{\mathrm{S}} ; r\right)=\frac{\alpha_{\mathrm{S}}}{\pi}\left[T_{t}^{2} \ln (1+r)+T_{u}^{2} \ln \frac{1+r}{r}+i \pi T_{s}^{2}\right]+\sum_{n=2}^{\infty}\left(\frac{\alpha_{\mathrm{S}}}{\pi}\right)^{n} \Gamma^{(n)}(r) .
$$

The amplitude $\left|\mathscr{M}_{H}\right\rangle$ depends on the flavour, colour and kinematical variables of the tree-level elastic scattering process. It embodies the residual terms of $\Sigma_{N}^{\text {res }}$ that are constant, i.e. of $\mathscr{O}(1)$ and not logarithmically enhanced in the limit $N \rightarrow \infty$. We write

$$
\left|\mathscr{M}_{H}\right\rangle=\alpha_{\mathrm{S}}\left(\mu_{R}^{2}\right)\left[\left|\mathscr{M}^{(0)}\right\rangle+\frac{\alpha_{\mathrm{S}}\left(\mu_{R}^{2}\right)}{2 \pi}\left|\mathscr{M}_{H}^{(1)}\left(\mu_{R}\right)\right\rangle+\sum_{n=2}^{\infty}\left(\frac{\alpha_{\mathrm{S}}\left(\mu_{R}^{2}\right)}{2 \pi}\right)^{n}\left|\mathscr{M}_{H}^{(n)}\left(\mu_{R}\right)\right\rangle\right]
$$

where we have omitted the explicit reference to the parton indices $a_{1} a_{2} a_{3} a_{4}$. By expanding the resummation formula in Eq. (3.4) to relative $\mathscr{O}\left(\alpha_{\mathrm{S}}\right)$ we can compare the logarithmic structure at this order with our general NLO result for Eq. (2.3). The structure of the logarithmic terms agrees and we can extract the NLO hard coefficient $\left|\mathscr{M}_{H}^{(1)}\right\rangle$. It contains the IR-subtracted NLO virtual corrections and is directly related to the process-dependent $\mathscr{C}_{1}$ function of Sect. 2 . Its expression is given in [22] Eq. (53), in terms of abstract colour-correlations operators. 
The all-order structure of Eqs. (3.4) leads to the exponentiated resummation of the logarithmic terms. After diagonalization $[7,29]$ of the anomalous dimension matrix $\Gamma\left(\alpha_{\mathrm{S}} ; r\right)$, the resummed radiative function of Eq. (3.4) can be written in the customary form [9, 21]

$$
\Sigma_{a_{1} a_{2} \rightarrow a_{3} a_{4}, N}^{\mathrm{res}}=\sum_{I} \widetilde{C}_{I, a_{1} a_{2} a_{3} a_{4}}\left(\alpha_{\mathrm{S}}\left(p_{T}^{2}\right)\right) \exp \left\{\mathscr{G}_{I, a_{1} a_{2} a_{3} a_{4}}\left(\alpha_{\mathrm{S}}\left(p_{T}^{2}\right), \ln N\right)\right\}+\mathscr{O}\left(\frac{1}{N}\right)
$$

where the index $I$ labels the colour-space eigenstates of $\Gamma\left(\alpha_{S} ; r\right)$, and $\widetilde{C}$ and $\mathscr{G}$ are functions of $\left(r ; p_{T}^{2}, \mu_{F}, \mu_{f}\right)$. The exponent function $\mathscr{G}_{I}$ includes all the $\ln N$ terms, while $\widetilde{C}_{I}$ includes all the terms that are $\mathscr{O}(1)$ in the large $N$ limit. The LL terms of $\mathscr{G}_{I}$ are controlled by the perturbative coefficient $A_{a}^{(1)}$, the NLL ones are determined by $A_{a}^{(2)}, B_{a}^{(1)}$ and $\Gamma^{(1)}$. The first-order term of $\widetilde{C}_{I}$ is computable from the explicit expression of $\left|\mathscr{M}_{H}^{(1)}\right\rangle$. Since we know $\Gamma^{(1)}$ and $\left|\mathscr{M}_{H}^{(1)}\right\rangle$, the colour interference between these two terms is known. The interference is taken into account by the correlated dependence on $I$ between $\widetilde{C}_{I}^{(1)}$ and $\Gamma_{I}^{(1)}$ in the exponent $\mathscr{G}_{I}$. The complete explicit determination of the NNLL terms in $\mathscr{G}_{I}$ still requires the coefficient $A_{a}^{(3)}$ (given in [33]), the coefficient $B_{a}^{(2)}$ and the second-order anomalous dimension $\Gamma^{(2)}$. The bulk of the contributions to $\Gamma^{(2)}(r)$ is expected to be proportional to $\Gamma^{(1)}(r)[27,10]$ via the factor $K / 2$. The coefficient $B_{a}^{(2)}$ could be extracted from NNLL computations of related processes, such as DIS [3].

\section{Summary}

We have studied the single-particle inclusive cross section at large transverse momentum in hadronic collisions, in the threshold limit in which the final-state system that recoils against the triggered parton is constrained to have a small invariant mass. In this case the accompanying QCD radiation is forced to be soft and/or collinear and the cancellation between virtual and real infrared singular contributions is unbalanced, leading to large logarithmic terms in the coefficients of the perturbative expansion. Using soft and collinear approximations of the relevant five-parton matrix elements, we have computed the general structure of these logarithmically-enhanced terms in colour space at NLO. The result of this NLO computation agrees with previous (colour summed) results in the literature, and it is presented here in a compact and process-independent form. This form is factorized in colour space and this allows us to explicitly disentangle colour interference effects. We have then presented the resummation formula (3.4) that controls these contributions to the $p_{T}$-dependent cross section at fixed rapidity. The formula, which is valid at arbitrary logarithmic accuracy, is written in terms of process-independent radiative factors and of a colour-space radiative factor that takes into account soft-gluon radiation at large angles. All the radiative factors are explicitly given up to NLL accuracy. Our process-independent NLO result agrees with the expansion of the resummation formula at the same perturbative order, and it allows us to extract the explicit form of the (IR finite) hard-virtual amplitude $\left|\mathscr{M}_{H}^{(1)}\right\rangle$ at relative $\mathscr{O}\left(\alpha_{\mathrm{S}}\right)$. The colour interference between this one-loop amplitude and the NLL terms explicitly determines an entire class of resummed contributions at NNLL accuracy. These resummation results are valid for both spin-unpolarized and spin-polarized hard scattering [22].

\section{References}

[1] G. F. Sterman, Nucl. Phys. B 281 (1987) 310. 
[2] S. Catani and L. Trentadue, Nucl. Phys. B 327 (1989) 323; S. Catani and L. Trentadue, Nucl. Phys. B 353 (1991) 183.

[3] A. Vogt, Phys. Lett. B 497 (2001) 228.

[4] S. Catani, D. de Florian and M. Grazzini, JHEP 0105 (2001) 025; S. Catani, D. de Florian, M. Grazzini and P. Nason, JHEP 0307 (2003) 028.

[5] N. Kidonakis and G. F. Sterman, Phys. Lett. B 387 (1996) 867, Nucl. Phys. B 505 (1997) 321.

[6] R. Bonciani, S. Catani, M. L. Mangano and P. Nason, Nucl. Phys. B 529 (1998) 424 [Erratum-ibid. B 803 (2008) 234].

[7] N. Kidonakis, G. Oderda and G. F. Sterman, Nucl. Phys. B 531 (1998) 365.

[8] E. Laenen, G. Oderda and G. F. Sterman, Phys. Lett. B 438 (1998) 173.

[9] S. Catani, M. L. Mangano and P. Nason, JHEP 9807 (1998) 024.

[10] R. Bonciani, S. Catani, M. L. Mangano and P. Nason, Phys. Lett. B 575 (2003) 268.

[11] G. F. Sterman and W. Vogelsang, JHEP 0102 (2001) 016.

[12] P. Bolzoni, S. Forte and G. Ridolfi, Nucl. Phys. B 731 (2005) 85.

[13] N. Kidonakis and V. Del Duca, Phys. Lett. B 480 (2000) 87.

[14] T. Becher, C. Lorentzen and M. D. Schwartz, Phys. Rev. Lett. 108 (2012) 012001.

[15] D. de Florian, A. Kulesza and W. Vogelsang, JHEP 0602 (2006) 047.

[16] L. G. Almeida, G. F. Sterman and W. Vogelsang, Phys. Rev. D 78 (2008) 014008; M. Czakon, A. Mitov and G. F. Sterman, Phys. Rev. D 80 (2009) 074017; V. Ahrens, A. Ferroglia, M. Neubert, B. D. Pecjak and L. L. Yang, JHEP 1009 (2010) 097, JHEP 1109 (2011) 070.

[17] W. Beenakker, T. Janssen, S. Lepoeter, M. Krämer, A. Kulesza, E. Laenen, I. Niessen, S. Thewes and T. Van Daal, report NIKHEF-2012-016 (arXiv:1304.6354 [hep-ph]).

[18] N. Kidonakis, Phys. Rev. D 81 (2010) 054028, Phys. Rev. D 83 (2011) 091503; H. X. Zhu, C. S. Li, J. Wang and J. J. Zhang, JHEP 1102 (2011) 099; J. Wang, C. S. Li and H. X. Zhu, Phys. Rev. D 87 (2013) 034030.

[19] S. Catani, M. L. Mangano, P. Nason and L. Trentadue, Nucl. Phys. B 478 (1996) 273; N. Kidonakis, G. Oderda, and G. F. Sterman, Nucl. Phys. B 525 (1998) 299; D. de Florian and W. Vogelsang, Phys. Rev. D 76 (2007) 074031.

[20] L. G. Almeida, G. F. Sterman and W. Vogelsang, Phys. Rev. D 80 (2009) 074016; R. Kelley and M. D. Schwartz, Phys. Rev. D 83 (2011) 045022.

[21] D. de Florian and W. Vogelsang, Phys. Rev. D 71 (2005) 114004; D. de Florian, W. Vogelsang and F. Wagner, Phys. Rev. D 76 (2007) 094021, Phys. Rev. D 78 (2008) 074025.

[22] S. Catani, M. Grazzini and A. Torre, Nucl. Phys. B 874 (2013) 720.

[23] R. K. Ellis, M. A. Furman, H. E. Haber and I. Hinchliffe, Nucl. Phys. B 173 (1980) 397.

[24] F. Aversa, P. Chiappetta, M. Greco and J. P. Guillet, Phys. Lett. B 210 (1988) 225.

[25] F. Aversa, P. Chiappetta, M. Greco and J. P. Guillet, Nucl. Phys. B 327 (1989) 105.

[26] S. Catani and M. H. Seymour, Nucl. Phys. B 485 (1997) 291 [Erratum-ibid. B 510 (1998) 503]. 
[27] S. Catani, Phys. Lett. B 427 (1998) 161.

[28] R. K. Ellis and J. C. Sexton, Nucl. Phys. B 269 (1986) 445.

[29] Y. .L. Dokshitzer and G. Marchesini, JHEP 0601 (2006) 007.

[30] P. Aurenche, R. Baier, A. Douiri, M. Fontannaz and D. Schiff, Nucl. Phys. B 286 (1987) 553.

[31] P. Aurenche, A. Douiri, R. Baier, M. Fontannaz and D. Schiff, Phys. Lett. B 140 (1984) 87;

P. Aurenche, R. Baier, M. Fontannaz and D. Schiff, Nucl. Phys. B 297 (1988) 661.

[32] L. E. Gordon and W. Vogelsang, Phys. Rev. D 48 (1993) 3136.

[33] S. Moch, J. A. M. Vermaseren and A. Vogt, Nucl. Phys. B 688 (2004) 101, Nucl. Phys. B 691 (2004) 129. 